\title{
Fonctionnement de l'usine de la Rance Comportement du matériel électromécanique
}

\author{
par MM. J. Lefrancois et Y. Marolleau \\ Ingénieurs à Electricité de France.
}

Après de nombreuses études dans les domaines les plus divers et six années de construction, le premier groupe de l'usine marémotrice de la Rance fut mis en service le 19 août 1966 et la dernière machine couplée au réseau le 4 décembre 1967. Ainsi s'achevait la construction de la première usine marémotrice du monde, avec seulement un retard de trois mois sur un programme de sept ans. Qu'est devenue cette réalisation originale après six ans d'exploitation? Depuis la fin de la période de mise aut point qui suit tout démarrage, l'usine fonctionne régulièrement, transformant le flux et le reflux de la marée en énergie électrique, comme l'avait imaginé ceux qui sont à l'origine de cette réalisation.

Avant de présenter le bilan de six années d'exploitation, nous rappellerons brièvement les caractéristiques des groupes de la Rance, leur intégration dans l'usine et les mises au point principales faites au cours de la période di démarrage. Nous examinerons ensuite le fonctionnement général et le comportement du matériel électromécanique en laissant de cóté les problèmes liés à la corrosion qui seront traités au cours de conférences distinctes.

\section{1. - Caractéristiques des groupes de la Rance}

L'exploitation de l'usine de la Rance exige le fonctionnement des groupes, comme des vannes, aussi bien au remplissage qu'à la vidange du bassin. Comme les cycles d'exploitation utilisent le pompage, les machines doivent pouvoir fonctionner en turbo alternateur et en moto pompe. Si l'on ajoute la marche non couplée en hyposynchronisme

dite «marche orifice »utilisée pour accélérer les opérations de vidange ou de remplissage du bassin, on obtient pour les mêmes machines six types de fonctionnements possibles:

turbine directe ) vidange du bassin machine couplée au pompe inverse réseau;

orifice direct : vidange du bassin machine non couplée; turbine inverse / remplissage du bassin machine couplée pompe directe au réseau;

orifice inverse : remplissage du bassin machine non couplée.

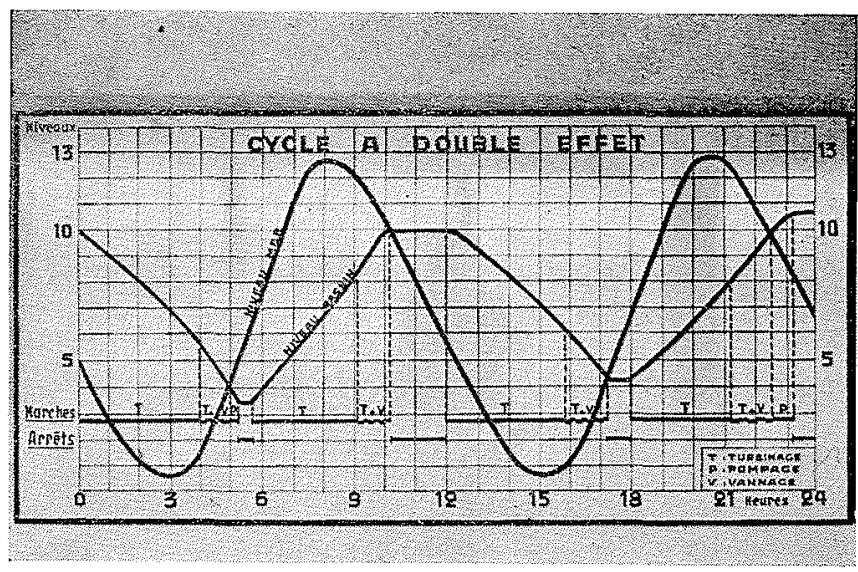

1/ Diagramme d'un cycle d'exploitation à double effet 


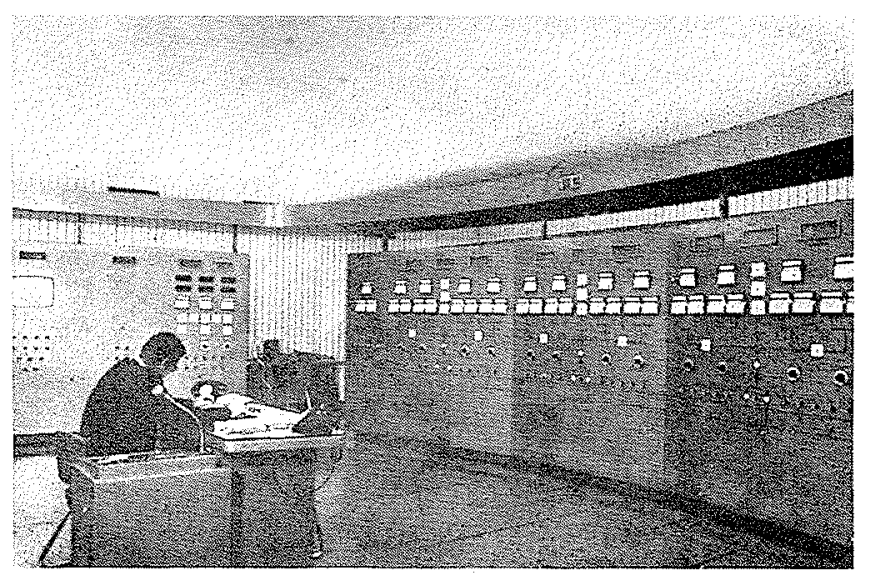

2/ La salle de commande centralisée

Pour exécuter cas différentes fonctions, il a fallu concevoir des groupes et un appareillage particuliers :

- Le sens de rotation des machines étant associé aul sens de l'écoulement de l'eau, un inverseur électrique permute deux phases du stator des alternateurs à chaque changement de type de marche couplée (directe ou inverse).

- Le démarrage en moteur à pleine tension, lors du fonctionnement en pompe a demandé un renforcement des caractéristiques asynchrones des machines : surdimen- sionnement de la cage d'amortisseurs, et construction d'un entrefer réduit à $5 \mathrm{~mm}$; il a nécessité aussi l'installation de disjoncteurs $3500 \mathrm{~V}$ ayant un pouvoir de fermeture de $170 \mathrm{kA}$ en crête et un pouvoir de coupure de $60 \mathrm{kA}$.

Rappelons que ces machines de $10 \mathrm{MW}$ sont regroupées par quatre, constituant ainsi une unité d'exploitation appelée « ensemble ». Chaque ensemble possède une centrale oléopneumatique et un système d'excitation communs aux quatre machines; il débite sur l'un des deux primaires d'un transformateur de bloc $3,5 / 225 \mathrm{kV}$ de $80 \mathrm{MVA}$

\section{2. - Mises au point au démarrage}

La mise en service de ces groupes particuliers fit apparaître trois difficultés importantes : l'une intéressait le joint d'étanchéité de l'arbre, les deux autres les rotors des alternateurs.

\subsection{Joint d'étanchéité de l'arbre (voir fig. 3, rep. 1).}

Le démarrage des premières machines fut marqué par de sérieux incidents sur le joint d'étanchéité radial de l'arbre, appelé aussi « joint carbone » car il est constitué par quatre couronnes de seize segments de carbone entourant l'arbre de la turbine. La couronne $\mathrm{n}^{\circ} 4$ située côté air, s'usait d'une manière inadmissible. Après de nombreux essais, une technique fut mise au point empiriquement; la lubrification, la création du film d'eau et la diminution de l'effort de ser-
3/ Coupe simplifiée de l'ensemble d'un groupe bulbe

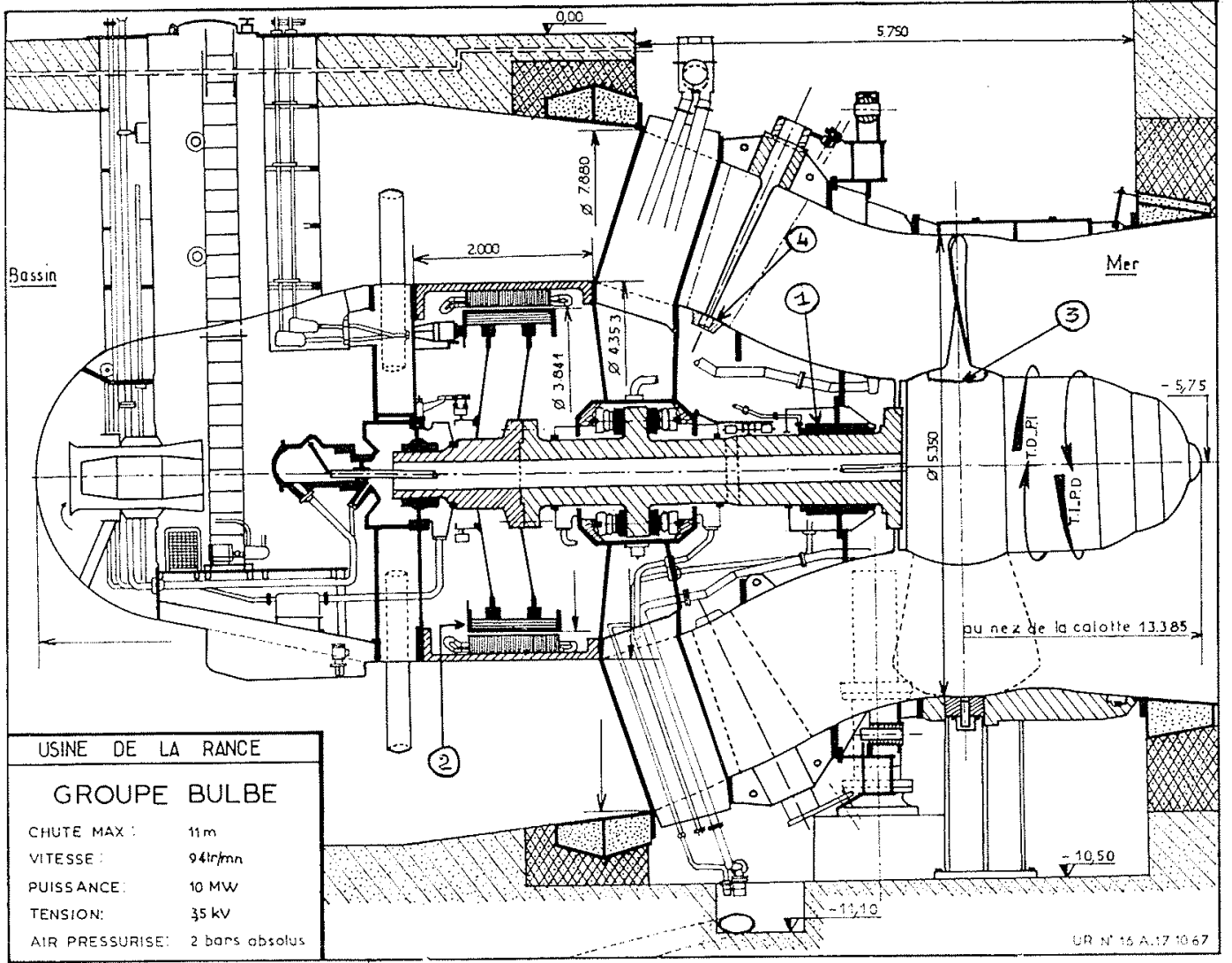




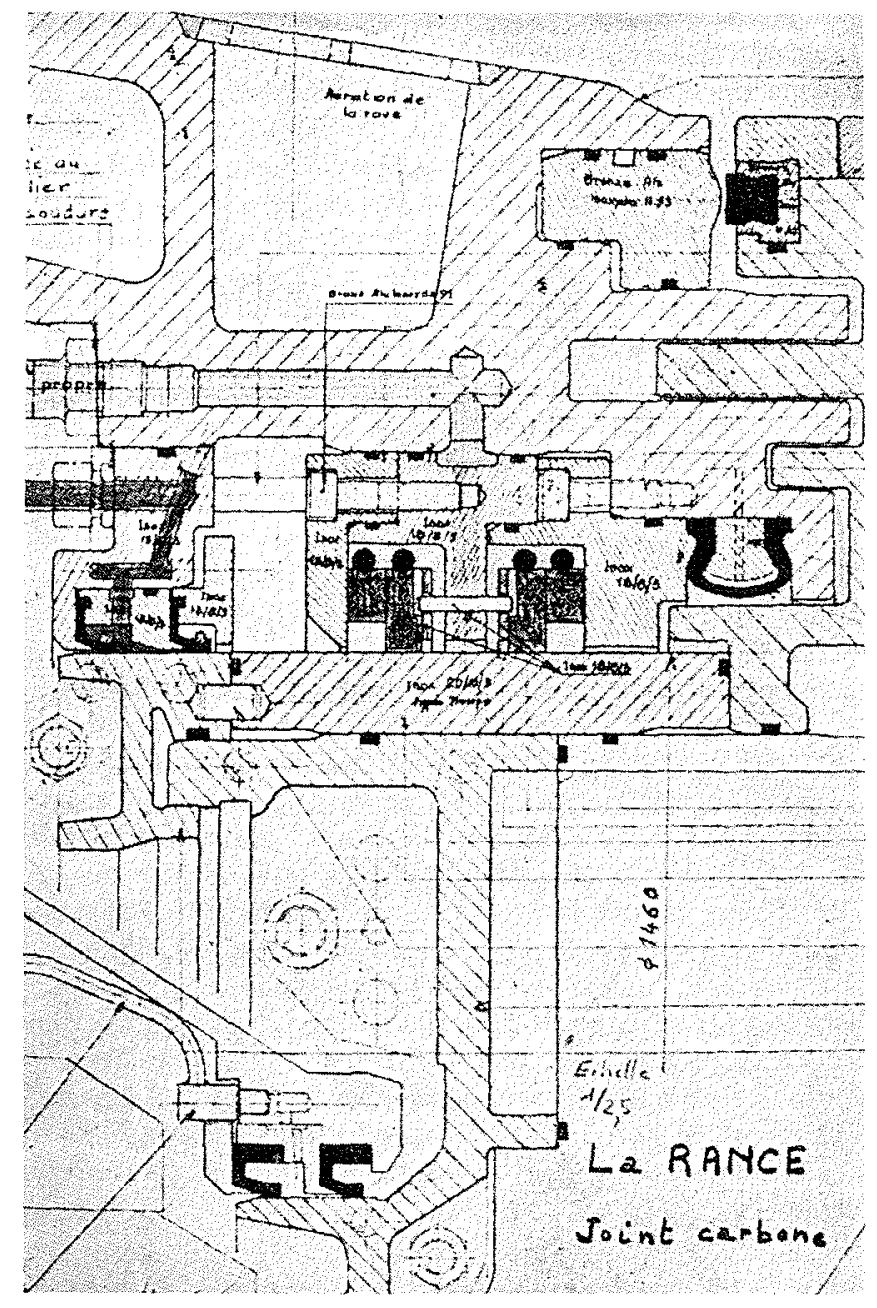

4/ Détail en coupe du joint d'arbre

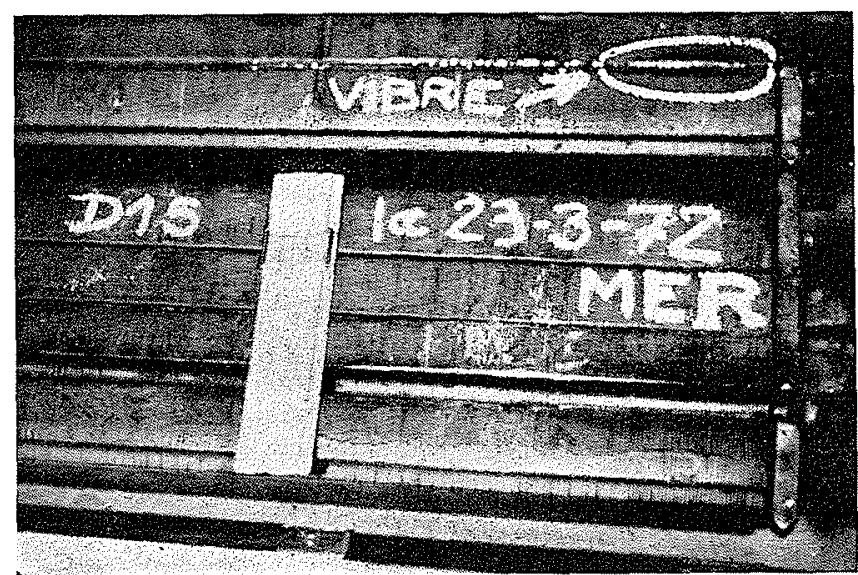

$5 /$

6/

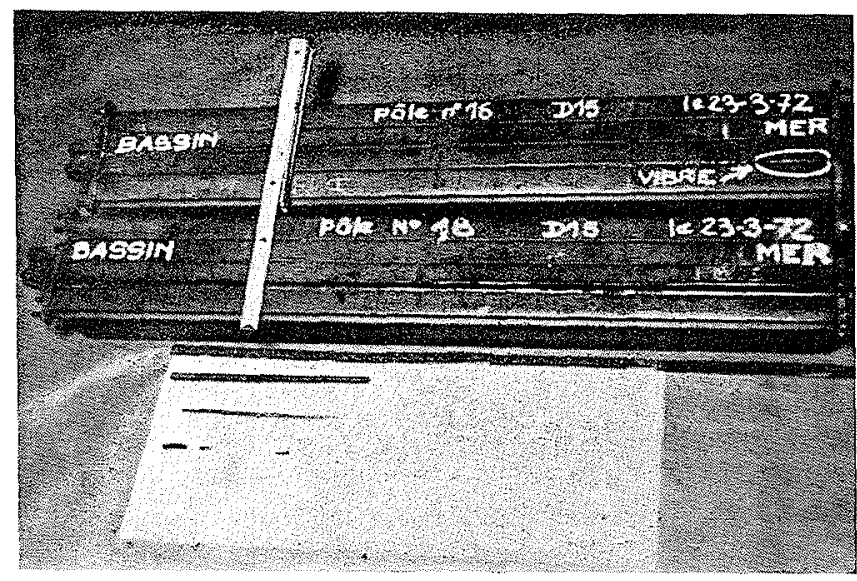

5 ef 6/ Pôles d'inducteur endommagés par électroérosion du fer autour des barres d'amortisseurs rage sur la couronne $\mathrm{n}^{\circ} 4$ furent favorisées par la création d'un chanfrein et de deux bassins d'amenée d'eau usinés, sur la face d'appui placée en regard de l'arbre. Depuis, dix-huit groupes ont été équipés selon cette solution, totalisant près de $500000 \mathrm{~h}$ de marche au $1^{\text {cr }}$ juillet 1972 , sans incident dû à une usure anormale; six autres groupes sont équipés, pour la 4" couronne, d'éléments en PTEF (teflon) chargé à $25 \%$ de carbone.

\subsection{Rotors des alternateurs (voir fig. 3, rep. 2).}

Le premier incident sur les rotors d'alternateur, fut découvert après les essais de survitesse à la suite d'une marche prolongée; on observa alors que le rotor, à jante flottante, avait légèrement touché le stator; cette anomalie était due à une dilation différentielle jante - bras du rotor plus forte que celle prévue par le calcul. Des cales furent placées entre la jante et les bras pour augmenter le serrage et l'épaisseur équivalente à ces cales fut enlevée sous les pôles du rotor.

A la suite d'incidents constatés sur les amortisseurs des pôles des machines de Pierre-Bénite, des investigations furent faites sur les rotors des alternateurs de la Rance. On releva un début d'usure des pôles par électro-érosion, provoqué par les courants induits dans les amortisseurs et dus aux harmoniques engendrés dans le stator. Les 64 pôles des 24 alternateurs furent démontés et les barres d'amortisseur matées pour éviter l'électro-étincellage.

Si le premier problème peut être considéré comme réglé à condition de ne pas atteindre la survitesse maximale exceptionnelle (événement de probabilité presque nulle), la tenue des barres d'amortisseurs devra continuer à faire l'objet de contrôles, car ces organes sont très sollicités lors des fonctionnements asynchrones.

\section{3. - Fonctionnement de l'usine}

Nous allons maintenant examiner le fonctionnement général de l'usine et rappeler les résultats de quelques essais effectués sur les machines. 


\subsection{Gencralitos.}

Si l'on croit certaines rumeurs aussi tenaces que fantaisistes qui courent dans la région malouine et même au-delà, l'usine de la Rance ne marche pas : un seul groupe fonctionne et le reste de l'installation est ensablé.

Evidemment, seules les aspirations des groupes $n^{\circ} 1$ et 24 sont couronnées par un vortex créé par la conjonction de leur position extrême avec un courant traversier; mais il suffit de regarder les remous à la sortie des machines pour rétablir la vérité, même sans être un spécialiste.

Quant au phénomène de l'ensablement des conduits hydrauliques il est sorti d'un esprit imaginatif et peu familier avec les notions d'hydrauliques, mais n'a pas atteint nos machines.

Si l'on revient aux données sérieuses, il faut retenir le taux d'indisponibilité des groupes en 1971:5\% dont 1,5\% seulement sur incident.

Si comme toute installation, l'usine de la Rance a connu des défaillances ou des incidents, nous y reviendrons dans la dernière partie de cet exposé; ils ne sont pas plus nombreux qu'ailleurs rapportés à la quantité de matériel installé bien que machines et appareillages soient nettement plus sollicités. Rappelons que les groupes démarrent en moyenne quatre fois par jour, alors que dans le même temps les disjoncteurs comme les vannes mancuvrent en moyenne trois fois. Le couplage des alternateurs, autorisé depuis l'arrêt jusqu'à $110 \%$ de la vitesse nominale, est sensiblement plus contraignant qu'une synchronisation classique. Malgré cela, depuis plusieurs années, l'usine fonctionne régulièrement, conduite et surveillée par un petit calculateur qui exécute le programme élaboré par le C.I.M.E. Ouest.

Pour mieux situer le fonctionnement des groupes, nous pouvons donner quelques chiffres :

Au ler juillet 1972, le temps cumulé total de marche des machines était de $620000 \mathrm{~h}$, soit une moyenne de $25800 \mathrm{~h}$ par groupe et qui se décomposait ainsi :

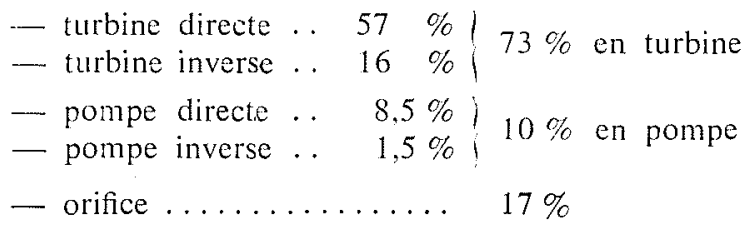

Le temps d'immersion maximal était de $51500 \mathrm{~h}$, alors que le temps moyen atteignait $45000 \mathrm{~h}$.

\subsection{Résultats des essais.}

De nombreux essais ont été réalisés, tant sur modèle que sur le site et il n'est pas possible de les résumer tous ici, même succinctement, d'autant que nombre d'entre eux ont fait l'objet de communications auprès de la S.H.F. Nous en citerons deux qui ont contribué à l'étude des groupes buibes, à savoir :

- les mesures de déformation, de contraintes, de vibrations d'une pale;

- le contrôle des échauffements, dus aux courants harmoniques dans un pôle de rotor.

Ensuite, nous évoquerons une étude particulière portant sur le couple hydraulique s'exerçant sur la roue, pales fermées, étude qui donna lieu à des essais sur modèle et des contrôles sur les machines.
La première série d'essais, préparés sur modèle, a permis de contrôler sur une pale en bronze d'aluminium du groupe A.3, la validité des calculs du taux de contrainte en pied da pale, taux qui reste inférieur à 14 hbar en régime permanent d'exploitation. L'analyse du spectre des vibrations a mis en lumière une oscillation basse fréquence due à l'apparition de la cavitation sur la face bassin lorsque la pale est au point haut, ainsi que des vibrations à des fréquences allant de 30 à $1000 \mathrm{~Hz}$ sans qu'aucune n'engendre des réactions dangereuses (la composante ondulée de la contrainte peut atteindre $10 \%$ de la contrainte moyenne en turbine inverse). Lors des déclenchements, les contraintes se situent assez loin de la limite de fatigue admise pour le matériau immergé, cela malgré les fluctuations importantes lorsque la fermeture des pales ramène la section de passage de l'eau à sa valeur minimale.

A la suite des incidents d'amortisseurs des rotors, une campagne d'essais fut lancée pour déterminer les échauffements de ces barres, tant au démarrage qu'en régime permanent; une analyse du flux superficiel et du courant dans les barres d'amortisseurs a complété ces essais. Pour le démarrage en pompe on a relevé un échauffement maximal des barres de $87^{\circ} \mathrm{C}$ alors que le fer du pôle ne s'échauffait que de $4{ }^{\circ} \mathrm{C}$. En régime permanent la température d'une barre s'est élevé à $144^{\circ} \mathrm{C}$ après une marche de deux heures à plus de 9 MW. Ces valeurs peuvent être considérées comme normales. (Lors de démarrages difficiles des échauffements de $200^{\circ} \mathrm{C}$ ont été mesurés). L'analyse des courants induits dans les barres fit apparaître la présence de forts courants de circulation avec un important pourcentage d'un courant à la fréquence $337,5 \mathrm{~Hz}$ qui correspond aux harmoniques d'encoches du stator. Ce phénomène est à l'origine des échauffements mesurés et de l'électro-étincellage constaté sur les encoches des barres d'amortisseurs.

Au cours des premiers démarrages en turbine directe sous forte chute, on observait le dévirage des groupes, pales complètement fermées. Puis, l'ouverture des pales provoquait le ralentissement, l'arrêt et le démarrage du groupe dans le sens direct. Des essais sur modèle montrèrent que le couple hydraulique était voisin du zéro et même négatif (inverse) lorsque les pales étaient complètement fermées. D'autre part, la machine modèle étant lancée, il fallait pratiquement fermer le distributeur pour la ramener au voisinage de la vitesse de synchronisme avec une chute supérieure à $6 \mathrm{~m}$.

Pratiquement on observe les phénomènes suivants :

- Pour les démarrages en turbine directe, il n'y a pas de difficultés majeures à condition de fermer le plus possible le distributeur avant le démarrage sous forte chute.

- Pour les démarrages en turbine inverse, on constate sur certains groupes un dévirage dans le sens direct de l'ordre de $30 \mathrm{tr} / \mathrm{mn}$ tant que les pales sont fermées. L'ouverture des pales amène le ralentissement, l'arrêt et la mise en vitesse dans le sens inverse des machines. Certains groupes ne démarrent pas et sont mis en route par le démarrage assisté.

- Le démarrage en pompe directe, donne lieu à un phénomène particulier. Pour les faibles chutes de l'ordre de $+0,50 \mathrm{~m}$, le groupe ne démarre pas. Mais lorsque la chute est positive, de l'ordre du mètre, l'eau en s'écoulant du bassin vers la mer entraîne la machine 
dans le sens de rotation inverse qui est celui de la pompe directe. Ainsi le groupe qui démarre hydrauliquement, est monté à la vitesse de synchronisme électriquement; c'est l'ouverture des pales qui provoque le renversement de l'écoulement de l'eau. Lors d'un essai en pompe directe sous $+3,30 \mathrm{~m}$ de chute, la vitesse dans le sens inverse des groupes de l'ensemble $\mathrm{E}$ était supérieure à $60 \mathrm{tr} / \mathrm{mn}$; le couplage a même ramené la vitesse du groupe E.19 de 100 à $93,75 \mathrm{tr} / \mathrm{mn}$.

\section{4. - Comportement du matériel électromécanique}

Nous allons maintenant examiner le comportement du matériel électro-mécanique en analysant les incidents les plus importants survenus depuis le démarrage sur trois ensembles des matériels suivants : les groupes, les vannes, et l'automatisme.

\subsection{Les groupes (voir fig. 3, rep. 3).}

La première défaillance sérieuse que nous avons constatée fut le défaut d'étanchéité des joints de pales de la roue. Ceux-ci, constitués par des joints chevron, sont concus pour éviter l'entrée de l'eau dans le moyeu de roue. Mais la présence de boue abrasive conjuguée avec un nombre important de manœuvres, avait fortement usé les lèvres de ces joints laissant l'eau de mer se mêler à l'huile de la roue. Pour éviter cette pénétration dangereuse, la pression de l'huile de compensation du moyeu de roue fut portée à une valeur supérieure à celle correspondant au niveau haut atteint par la mer et tous les joints furent remplacés sur les pales des vingt-quatre groupes. Un essai de joint torique en caoutchouc, actuellement en cours, donne des résultats intéressants; il sera sans doute souhaitable d'essayer un caoutchouc avec une dureté plus faible (55-60 shore au lieu de 65-70) pour accroître la sécurité de l'étanchéité. Malgré ces précautions, nous purgeons tous les ans le moyeu de roue de chaque groupe (voir fig. 3, rep. 4). Un deuxième problème relatif aux turbines a pour origine le gonflement de bagues Céloron dans l'eau.

\section{UBINEDELARANCE}

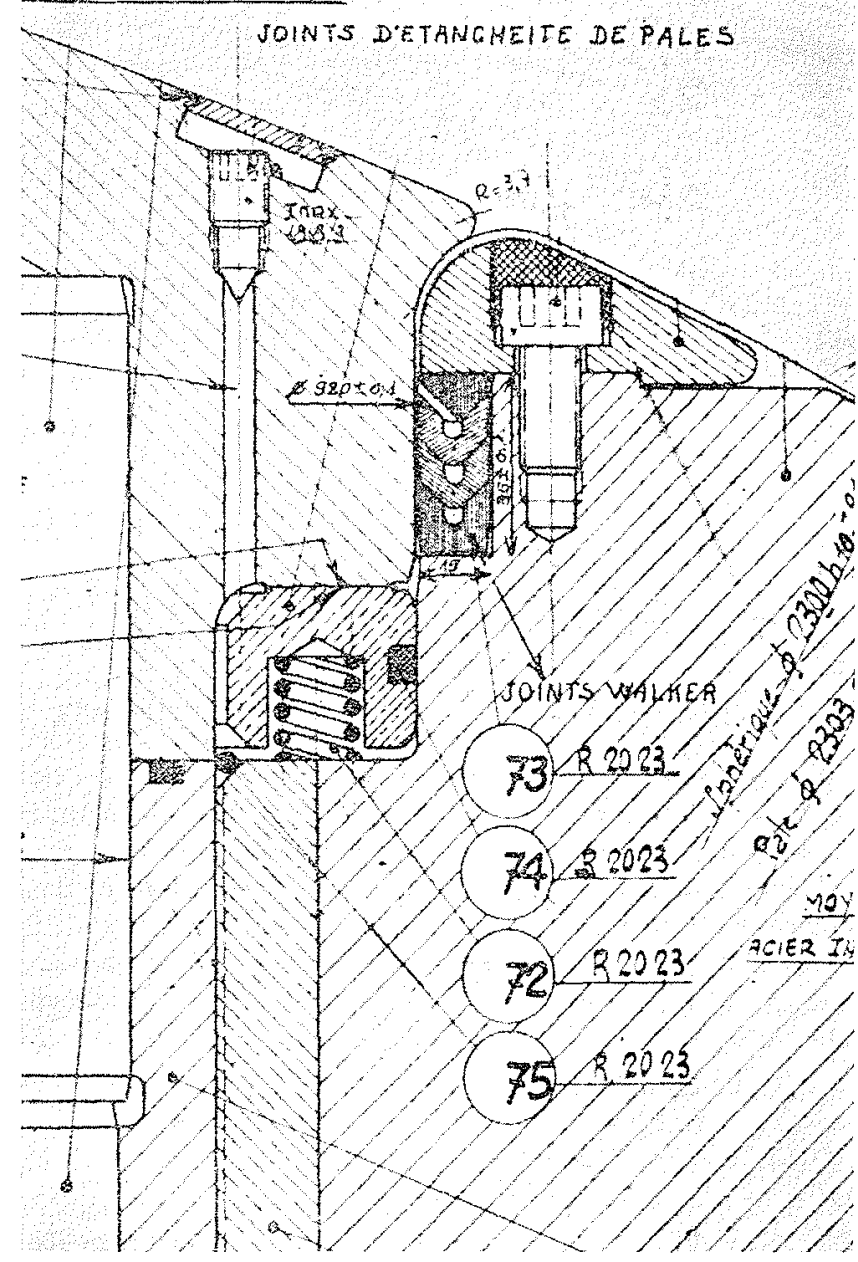

7/ Détail du joint cntre pied de pâle et moyeu du moulinet

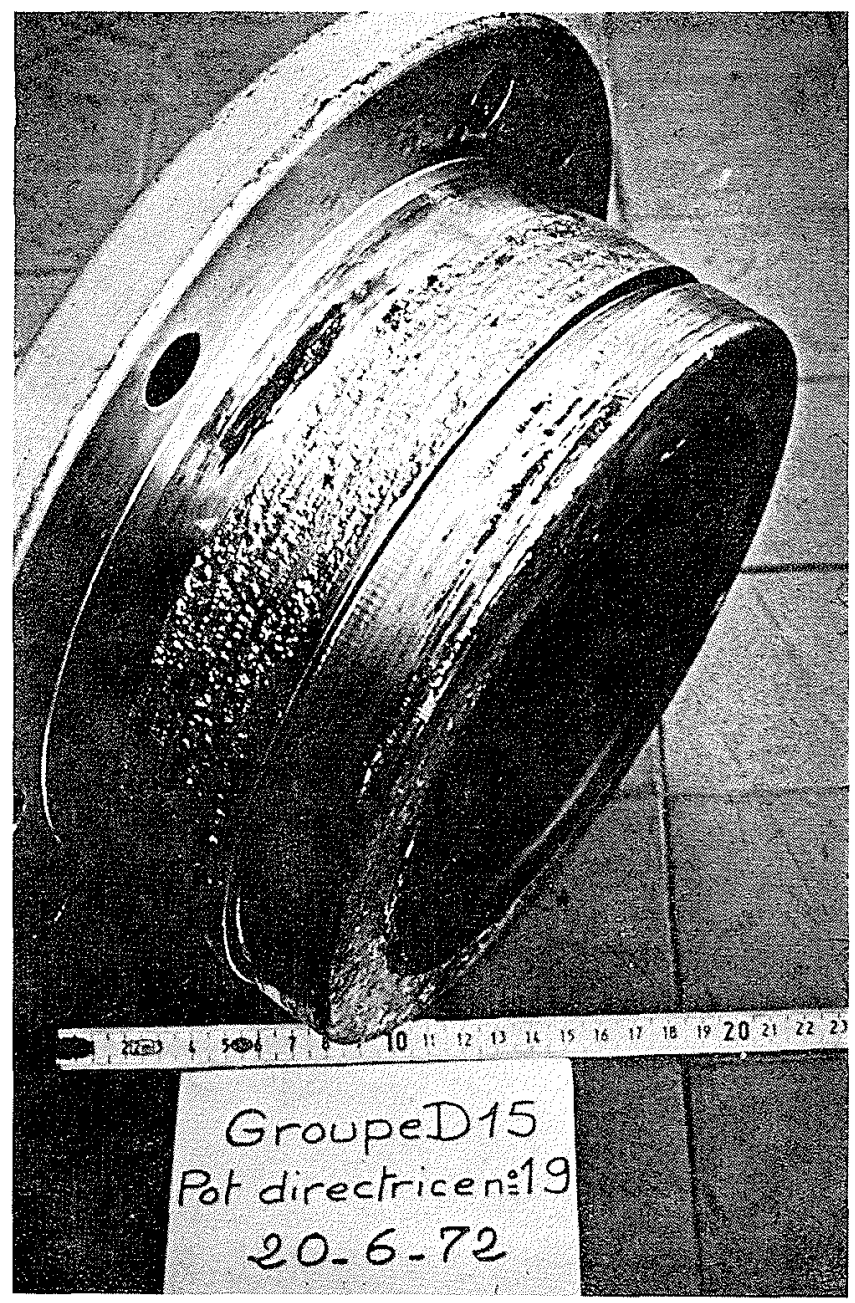

8/ Pot de directrice endommagé après gonflage du palier en céloron 
Ce phénomène est bien connu puisque le fournisseur préconise une immersion prolongée dans l'eau avant l'usinage définitif. Cette précaution n'a pas été suffisante pour les pots intérieurs des directrices, car nous avons constaté que la rotation des directrices avait entraîné le dévissage ou la rupture des vis de fixation de certains pots sur le distributeur. Nous avons mesuré jusqu'à $1 / 10 \mathrm{~mm}$ de serrage sur des pots démontés. Aussi, nous démontons les vingt-quatre pots de directrices sur chacun des groupes pour les réaléser, opération qui sera terminée au début de l'année 1973. Ce même phénomène de gonflement du Céloron a d'ailleurs été constaté sur les boîtards des galets des vannes de coupure.

Pour les alternateurs, nous avons rencontré deux problèmes: l'un concerne l'usure des balais des bagues du rotor et l'autre la qualité de l'isolement des stators.

La tenue des balais des bagues rotoriques est médiocre depuis la mise en service; la situation des bagues dans l'enceinte étanche pressurisée contenant des vapeurs de solvants libérées par les isolants et les vernis, le double sens de rotation, le fonctionnement sans courant pendant la marche en orifice entraînaient une usure importante des balais qui pouvait dépasser $10 \mathrm{~mm}$ en $1000 \mathrm{~h}$. De nombreuses qualités de charbon ont été essayées et actuellement, celles contenant une charge d'argent en quantité appréciable donnent des résultats acceptables : l'usure est pratiquement inférieure à $1 \mathrm{~mm}$ en $1000 \mathrm{~h}$ sur tous les groupes. La grande dispersion des résultats doit conduire à rester vigilant d'autant que certaines qualités tenclent à user les bagues.

Quant aux défauts d'isolement sur les stators, il se sont manifestés pour la plupart à l'occasion des contrôles périodiques, un seul groupe ayant claqué en marche; les défauts, d'origines diverses, sont causés soit par blessure des barres par des cales isolantes, soit par des manques de continuité dans l'isolement des développantes. Il faut d'ailleurs reconnatitre que la poussière de charbon est un très bon révélateur de ces défauts.

Si d'autres incidents sont moins gênants par les répercussions qu'ils entraînent, ils peuvent conduire à une maintenance importante compte tenu de la masse de matériel concerné. Il s'agit en particulier des organes fonction-

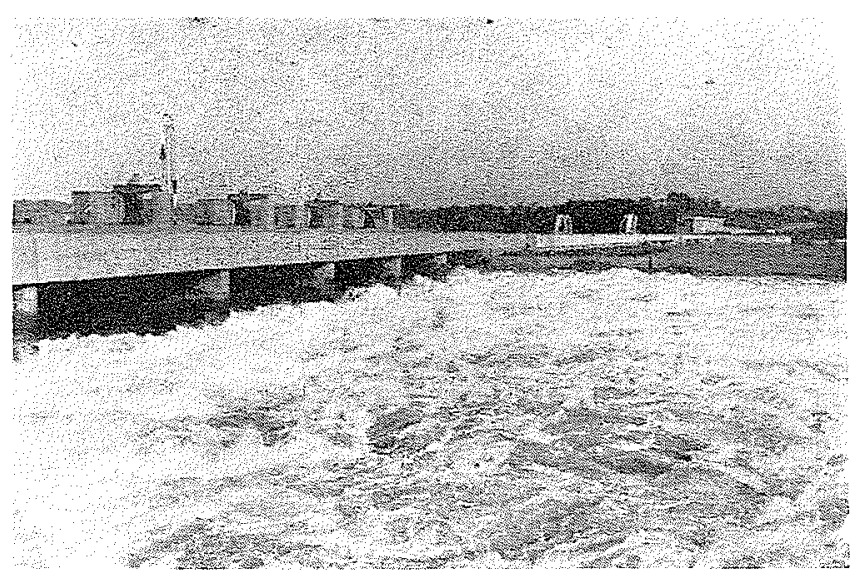

9/ Le flot ì la sortie des vannes ouvertes nant d'une manière intensive : électrovannes, actionneurs de régulation, pompes; de ceux qui, soumis à la corrosion, ne sont pas protégés cathodiquement comme le boîtier de joint carbone. Il faut aussi mentionner les difficultés constatées avec certains joints toriques d'étanchéité statique qui, suivant leur qualité et les conditions de travail maigrissent, ou durcissent, ou se décollent. Dans ce dernier domaine, citons la solution que nous avons mise en cuvre pour étancher lo raccordement distributeur - avantdistributeur. Sur deux groupes nous avions constaté des venues d'eau dans le circuit de graissage du palier turbine et de la butée et nous avons mis en lumière le contournement par l'eau de mer du joint torique d'assemblage placé entre les brides du distributeur et de l'avant-distributeur; comme il n'était pas question de remplacer ce joint, car cette opération aurait demandé plusieurs mois, nous avons procédé à des injections de résines acryliques dans la gorge du joint ce qui a permis d'étancher la fuite. Cette technique a été aussi adoptée pour arrêter les fuites d'huile au raccordement des deux parties de l'avant-distributeur formant corps de butée et réservoir d'huile de cette butée.

Pour en terminer avec les groupes, signalons la présence de balanes, petits coquillages tronconiques qui en se collant sur les pièces d'étanchéité des vannes de coupure, sur le béton et dans les tuyauteries de vidange rendent souvent difficiles la vidange des conduits hydrauliques. Des essais de peintures anti-salissures sont en cours actuellement.

\subsection{Vamnes.}

Les travaux de révision mécanique et de réfection de peinture des six vannes, commencés en 1968, se sont terminés en 1972. Dans l'ensemble ce matériel soumis à rude épreuve a bien tenu. L'incident le plus notable est dû au percement des tuyaux d'huile de graissage des galets, percement causé par la corrosion avant l'installation de la protection cathodique; cette défaillance du circuit de graissage a entraîné quelques usures tant sur les fusées que sur les coussinets des galets.

\subsection{Automatisme.}

Avant de conclure, nous voudrions dire quelques mots de l'automatisme de cette centrale.

En dépit d'un certain nombre de défaillances de relayage, bobines de contacteurs ou de relais détériorés, l'automatisme classique fonctionne bien surtout si l'on tient compte de la complexité imposée par le regroupement de quatre machines dans un "ensemble », des différents types de fonctionnement, de la fréquence de manouvre et de la quantité de matériel installé.

Pour faciliter les tâches de conduite et de surveillance, un petit calculateur pilote l'usine, assurant les tâches suivantes

- la fonction «Programmateur» qui permet d'exécuter la programme élaboré par le Centre Interrégional du Mouvement d'Energie « Ouest»;

- la fonction «Microrattrapage \& qui corrige l'incidence des pales des groupes pour tendre à retrouver en deux heures la chute prévue par le programme;

- la fonction «Déchargeur » qui calcule et affiche l'incidence " déchargeur » que prendraient les ensembles en cas de passage en marche en déchargeur; 


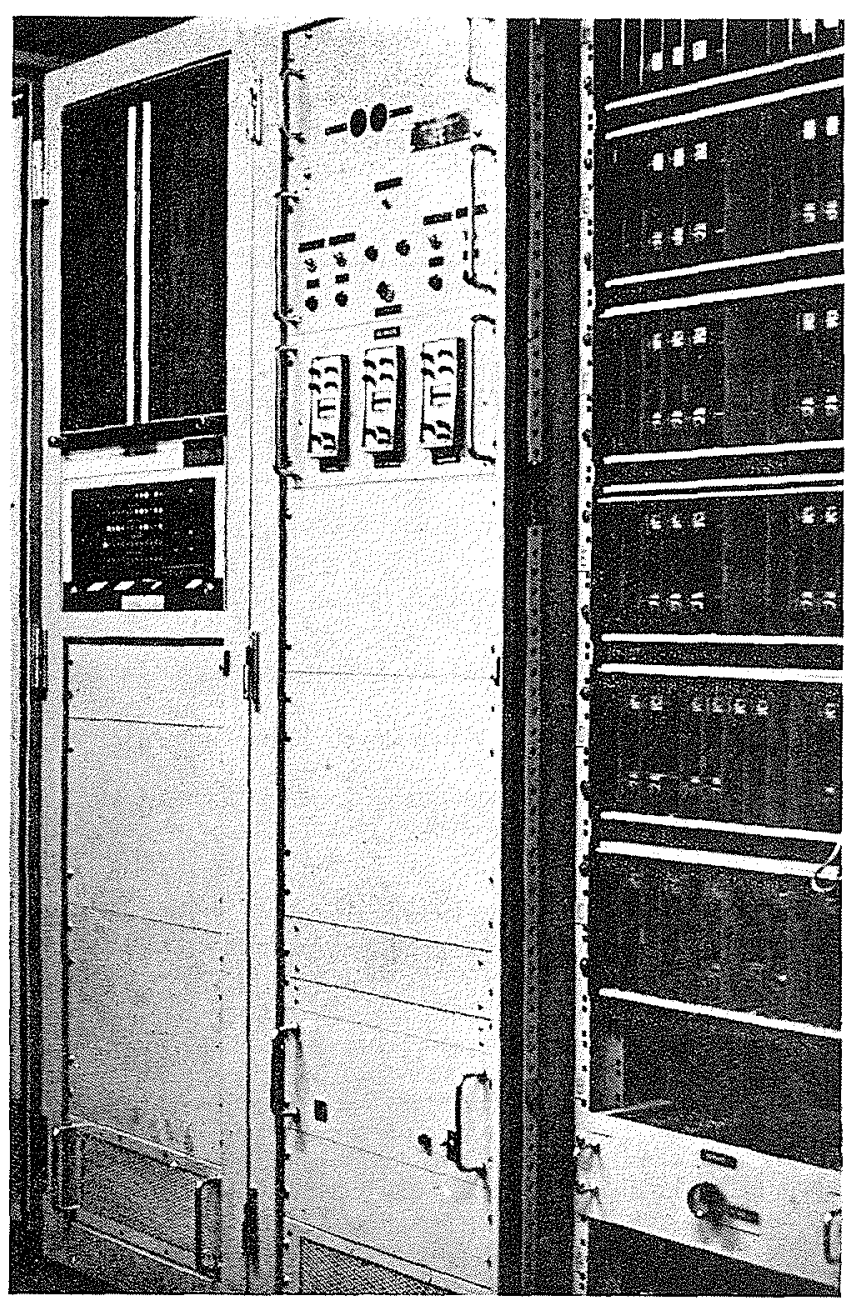

10/ Ensemble du calculateur

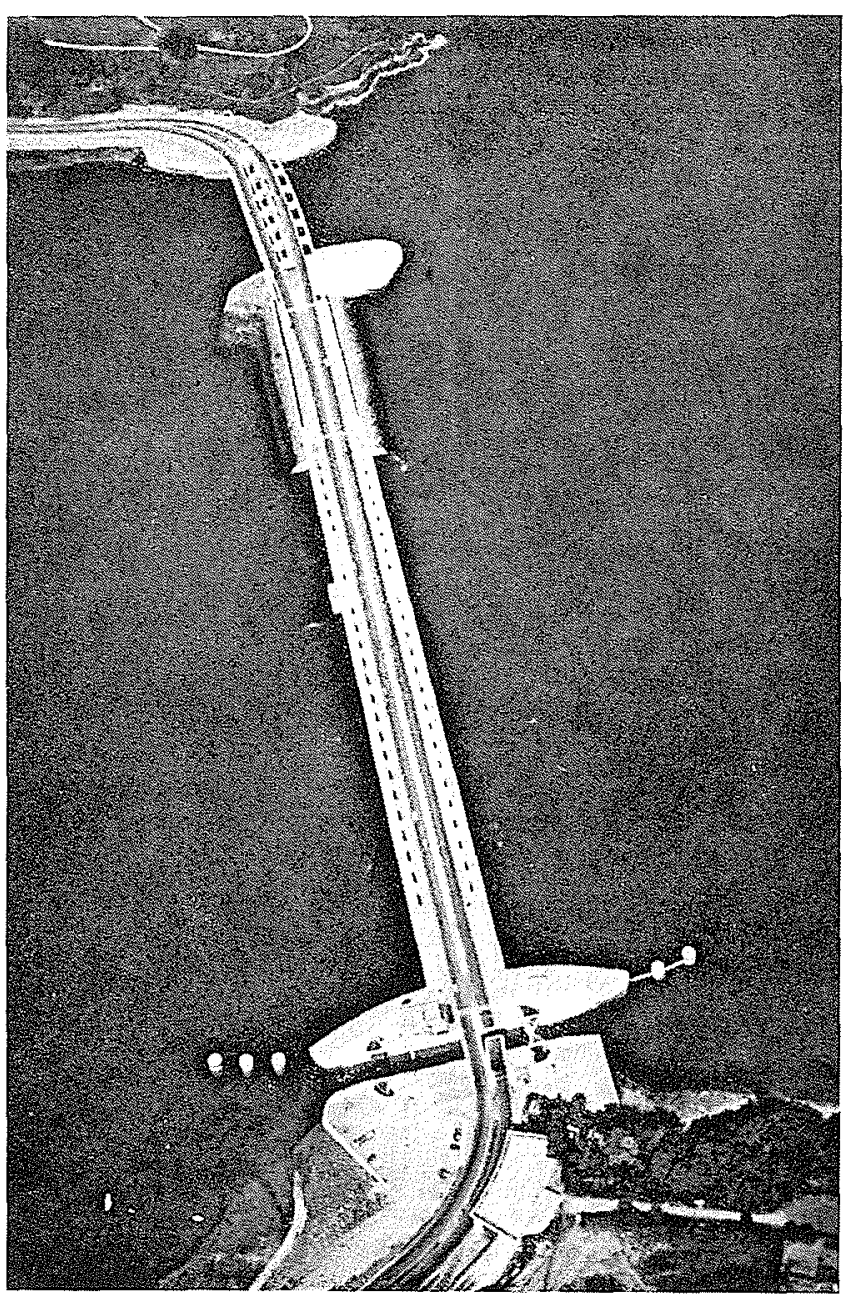

11/ Vue aérienne du barrage: «une règle posée sur l'eau».
- la fonction «Centralisation des défauts » qui exécute la scrutation des 1920 voies de défauts en fonctionnement cinquante fois par seconde et imprime les changements d'état entre deux scrutations consécutives.

Après une mise au point assez longue causée par des difficultés dues à des inductions parasites, cet ensemble fonctionne parfaitement, et en un an et demi, nous n'avons connu qu'une seule panne, sur l'unité centrale et les interfaces, qui nous a obligé à remplacer un module. Ainsi, lusine de la Rance a été la première centrale hydraulique française et peut-être même au monde, à être automatiquement conduite et surveillée par calculateur.

\section{Conclusions}

Au moment de l'inauguration, il y a six ans, le Président de la République écrivait sur le livre d'or de l'usine « Bonne chance à l'usine marémotrice de la Rance $»$. Il est probable que ceux qui vivaient à cette époque les difficultés de mise au point des joints d'arbre, avaient besoin d'une certaine foi pour accepter ces vœux. Aujourd'hui, après six ans d'expérience, nous connaissons mieux l'ouvrage qui nous a été confié. Si cette usine fonctionne régulièrement, comme l'avait imaginé ceux qui l'ont conçue, elle le doit moins à la chance qu'à la valeur et l'audace des études dont elle fut l'objet, la qualité de la construction du génie civil, de la fabrication et du montage du matériel électromécanique et la continuité d'une maintenance qui cherche à prévenir les incidents.

Ils sont probablement peu nombreux ceux qui, parmi les millions de personnes qui empruntent chaque année la route du barrage, imaginent la somme de travail accumulé sous eux. Qui songe aux nombreuses études et essais, remodelós, affinés pour chercher à parvenir à l'optimum, à la construction du barrage, à la fabrication et au montage des machines, entreprise entourée des inconnues et des risques d'une première mondiale? Qui pense au travail obstiné de ceux qui quotidiennement doivent conserver l'intégrité de l'ouvrage tout en cherchant à lui faire rendre le maximum?

Diverses communications vous ont exposé ou vous 
exposeront les aspects positifs du barrage de la Rance, outre la production d'énergie électrique évidemment. Pour terminer, nous voudrions mentionner un mérite qui peut passer inaperçu à ceux qui n'ont pas vécu cette grande aveniure de la conception, de la construction ou de l'exploitation de l'usine de la Rance. Comme toute grande ouvre humaine, exigeante et passionnante, elle a demandé à ceux qui s'y sont consacrés, quelle que soit leur fonction, de donner le meilleur d'eux-même. Aussi, en plus d'un certain nombre d'apports bénéfiques, à travers les difficultés, les échecs et les succès, l'usine de la Rance a-t-elle contribué à former des techniciens, mais aussi des hommes.

\section{Discussion}

Président: M. X. Ract-Madoux

M. le Président remercie M. Lefrancois de son exposé qui reflète, dit-il, une expérience déjà longue des groupes bulbes; en effet, avant de s'occuper de l'usine marémotrice de la Rance et du sous-groupe d'Aurillac dont dépendait l'usine de Cambeyrac, notre conférencier avait eu la charge de l'exploitation des petites centrales de la BasseMaulde, qui totalisaient vingt-trois groupes bulbes.

Il ouvre ensuite la discussion.

M. Duport pose la question suivante:

Vous avez fait allusion aux efforts déployés par les services d'exploitation pour «maintenir en état de bon fonctionnement" l'usine et son équipement. Quels sont les effectifs y affectés et les charges financières correspondantes? Il nous a été indiqué que l'incidence de cette maintenance sur la disponibilité n'était que $1,5 \%$ - comparable aux usines classiques de basse-chute. En est-il de même pour la charge financière - en personnel, en particulier entraînée par cette mainterance?

\section{LEFRANÇOIS répond:}

Votre question, très pertinente, nous est souvent posée par des personnes bien informées des problèmes de la production hydraulique. Les difficultés inhérentes à un groupe ne sont pas, je crois, très supérieures sur la Rance à celles qu'on rencontre dans une usine de basse-chute. Elles le sont légèrement pourtant du fait de l'eatl de mer, de la corrosion et des cycles imposés aux machines qui supportent des démarrages et des arêts plusieurs fois par jour.

La difficulté vient du fait qu'il y a vingt-quatre «machines». Une usine équivalente, de même puissance, avec huit groupes de $30 \mathrm{MW}$, donnerait beaucoup moins de travail qu'une usine de vingtquatre groupes de $10 \mathrm{MW}$.

J'ai évoqué le problème de réalésage des coussinets de directrices: c'est une opération que nous avons décidé d'entreprendre fin 1971. Nous en avons établi la programmation de manière à éviter des indisponibilités anormales en période de haute mer. C'est un programme qui s'étend sur dix-huit mois et se terminera au début de 1973.

Nous savons ainsi que la moindre réparation qui s'est imposée surr un groupe va se répercuter sur les vingt-quatre groupes de l'usine ct représente un travail de dix-huit mois.

C'est le fait du nombre de machines qui rend l'affaire lourde. (C'est cela qui nécessite la présence d'un personnel relativement mmportant. L'équipe globale à l'usine de la Rance est composée en majorité de personnel de conduite et de personnel d'entretien. Elle somporte cinquante personnes.

M. Lefrançors serait-il candidat pour être directeur de la première usine de séparation isotopique qui va compotter plusieurs centaines de centrifugeuses marchant toutes à 120 ou 130000 tours, demande M. Gibrat?

Non pas spécialement, répond M. LefrançoIs, parmi les rires de l'assistance.

M. Cazenave (Compagnie Nationale du Rhône) intervient en ces termes :

L'usine marémotrice peut conduire à des défauts ou des difficultés plus grands qu'une usine en rivière, pour deux sortes de raisons principales :

- la nature de l'eau;

- la complexité des cycles de fonctionnement.

D'après votre expérience, quel est, de ces deux éléments, cclui qui vous parait le plus important?

M. LEFrançors répond :

Maintenant que les problèmes de corrosion ont été maitrisés, les difficultés essentielles ne proviennent pas de l'eau de mer mais du fait que les cycles de fonctionnement journaliers d'une usine marémotrice imposent au matériel des contraintes extrêmement sévères.

Alors que dans la mesure des débits disponibles, une Centrale implantée sur le Rhône ou sur le Rhin démarre un jour J et s'arrête sauf incident trois mois après ou quatre après, au contraire, les groupes de la Rance démarrent et s'arrêtent au plus tard $6 \mathrm{~h}$ après pour redémarrer $3 \mathrm{~h}$ après et s'arrêter $4 \mathrm{~h}$ plus tard, avec des inversions de sens de marche et des démarmages en moteur.

L'exploitation d'une usine marémotrice implique le fonctionnement en pompage; celui-ci nécessite des caractéristiques asynchrones sur les machines électriques, ce qui n'est pas tellement favorable à leur maintien en bonne santé. En particulier, l'échauffement des barres des cages d'amortisseurs ne peut passer inapercu.

$Y$ a-t-il des renseignements à tirer de la réversibilité des machines, notamment en ce qui concerne la tenue des balais? demande $M$. le Président.

Si l'on avait pu supprimer les balais en alimentant l'excitation des alternateurs par un redresseur statique toumant, là tâche des Services d'entretien êt été bien allégée, répond $M$. LEFRANÇors.

D'après les études effectuées par la Direction des Etudes et Recherches de IE.D.F., les difficultés rencontrées proviendraient moins de la réversibilité des machines que de leu fonctionnement en atmosphère confinée; les vapeurs de solvant, évaporées de l'isolation des bobinages polluent l'atmosphère intérieure de la machine; il en résulte parfois des résistances de contact inadmissibles entre bague et balai. On peut donc espérer que la situation s'améliorera avec le temps du fait de l'évaporation progressive desdits solvants.

En labsence d'autre intervention, M. le Professeur clot la discussion. 